\title{
Research on Finite Element Method Based on Point Model
}

\author{
Shengliang Zou ${ }^{1,{ }^{* a}}$, Shaojun $\mathrm{Yi}^{2, \mathrm{~b}}$ \\ ${ }^{1}$ Nanchang University of Gongqing College, Jiujiang City, China; \\ ${ }^{2}$ Nanchang University of Gongqing College, Jiujiang City, China. \\ *Corresponding author Email: ${ }^{\star} 731944618 @ q q . c o m,{ }^{b} 22756050 @ q q . c o m$
}

Keywords:Point model; Finite element algorithm; 3D modeling

\begin{abstract}
This paper mainly introduces the application of nonlinear finite element method in dynamic simulation based on point model. In this paper, based on the traditional nonlinear finite method, the method of optimizing the tetrahedral mesh is proposed, and the mathematical model and calculation method that can unify the elasticity and the large plastic flow are proposed.
\end{abstract}

\section{Introduction}

With the development of 3D modeling technology, static 3D models can no longer meet the needs of the actual market. For example, in virtual reality scenes, 3D animation production and computer aided design require realistic 3D dynamic images, especially simulation based on mathematical processes. Requires a rigorous theoretical basis based on mathematics, dynamics, thermal science, chemistry and other related disciplines[1]. For example, the simulation of hurricanes and tsunamis has an inestimable value for the national economy. This article is based on the static three-dimensional model data generated on the basis of the study of the dynamics of the real world, especially the deformation of the mathematical model and calculation methods, so that it can be easily expressed using computer graphics[2].

\section{Current research status}

Computer graphics-based virtual reality, visualization of scientific computing and computer simulation technology are currently hot topics in the academic community. Among them, modeling and simulation based on dynamic mathematical processes is one of the main research directions. In the processing of dynamic problems, the early method of using pure geometry, but it is difficult to obtain good results, and mathematical modeling methods not only make up for the lack of geometric modeling in the modeling process, in the interactive simulation process It also enhances the realism of the simulation.

Many researchers use particle-based methods to simulate high-viscosity fluids and fluids with elastic properties. Poulterer simulates melting thermodynamic materials. When the material is in a solid state, each particle is connected to adjacent particles in the form of an elastic spring. When the material has fluid properties, the elasticity becomes weak or even disappears[3]. By changing the elastic properties of the material, this method can simulate the behavior of a wide range of changing materials, but does not include plastic strains, so it cannot handle deformations similar to clay materials. Muller demonstrated that the computational results using the particle-spring model are comparable to current grid-based methods. Although these methods are currently mainly used to simulate the behavior of rigid fluids, it is difficult to extend them to other material models[4]. At present, the finite element method is the most widely used mechanical calculation method in the field of computer graphics simulation. In the simulation process, corresponding to the specific simulation object, the classic complete finite element method and the updated finite element method are correspondingly improved to adapt to the specific problem[5]. 


\section{Point model basis}

In the three-dimensional image processing and three-dimensional scanning technology, a large number of geometric models represented by points are directly generated. If a traditional surface-based method is used, these points need to be represented as a triangular mesh by a triangulation technique such as the Delaney method, and then Processing or display, this not only increases the amount of data, but also reduces processing efficiency. In order to further improve the processing and rendering efficiency of the geometric model, the point is regarded as a basic drawing element, so that the triangulation and subsequent triangular mesh representation and transmission are omitted, so that higher processing efficiency can be obtained. The use of points as representational elements of the model stems from the simple idea that a model of any shape can be represented by multiple points at its boundaries (surfaces)[6]. The quality of the representation depends only on the number of points and is not subject to topological relations. constraint. Unlike point cloud-based surface reconstruction, a point-based model representation uses a point as a basic shape to represent units and investigates related geometrical operations, while the former uses alternative representations instead of point representations[7].

In the point-based model representation method, the local information of a certain point is the most concern of people. Using these local information, some important geometric attributes of the point can be calculated, such as a normal vector. In addition, due to the large number of points in the point model, the efficiency of the local algorithm is much higher than that of the global algorithm. Although the point model-related algorithms are mostly local algorithms, we hope that all local calculation results are consistent with the global algorithm and a smooth two-dimensional manifold can be obtained.

The set of points can be regarded as a set of samples of the surface position of the unknown surface

$$
\begin{gathered}
\text { F, } P=\left\{P_{i} \in R^{3}\right\} \\
i \in\{1, \ldots m\} \\
N=\left\{n_{i} \in R^{3},\left\|n_{i}\right\|=1\right\}
\end{gathered}
$$

where, like the samples of other signals, there may be some noise therein and it is usually irregular. Some sampling procedures can also obtain the normal for each point on the surface, denoted as .

The purpose of the point model is to define the calculation of various operations on any point in the point set. These methods do not directly use the surface constructed from the point set through the global method, but use several points or adjacent areas related to the operation of the point. Calculation. If so, the surface is an interpolation surface, otherwise it is an approximate surface.

\section{Point model representation based on KD tree}

One of the characteristics of the point model is that it does not use the topological relationship between the recording points. The relationship between the points is completely determined by their spatial position. Therefore, some spatial data structures, such as the binary space partition tree (BSP tree), octree, KD tree (multidimensional binary tree) is very suitable for the representation of point models. A KD tree is a binary tree. Each node corresponds to a rectangular box and a dividing plane. The dividing plane is parallel to one coordinate axis. The dividing plane divides the rectangular box into two sub-cuboid boxes and forms two child nodes of a KD tree. For the point set, the selection method of the split plane determines the construction rules of the KD tree. According to the different construction rules, the KD tree can be divided into a midpoint split KD tree, an equalized KD tree and a sliding midpoint KD tree. The split plane of the midpoint split KD tree is located at the center of the cube corresponding to the node, and is parallel to the coordinate axis. This KD tree has a bit sharing feature between nodes of adjacent layers. The division plane of the balanced KD tree passes through one of all points included in the node, and makes the number of points on both sides of the division plane approximately equal. The sliding midpoint KD tree divides a node by moving the split plane 
located in the center of the cube to a point that is closest to it. In addition to splitting the KD tree at the midpoint, the other two KD trees do not have bit sharing features.

\section{Solution to Nonlinear Problems}

At present, there are two description methods to establish the nonlinear finite deformation of the finite element equations, a Lagrange description method, which is based on the initial state graphics description, also known as the particle coordinate system description; the other is Euler describes the method, which is described by reference to the distortion concept, and is also called the flow coordinate system description. The Lagrangian method is a relatively natural and effective description method and is usually used for the formulation of solid mechanics and structural mechanics. The Euler description method mainly analyzes the motion of a substance with a fixed control volume, as used to analyze large displacement structures. Because the new control volume must be constantly established, because its boundaries are constantly changing, it is not very convenient. This method is usually used to analyze fluid mechanics problems. The Lagrangian method fixes the origin of the coordinate to a certain point in the system. When the configuration of the system changes, the coordinates also move along with it. When using the Lagrangian method, the position of the node on the object is fixed, and the object unit is composed of nodes. When the object is deformed, the node moves with the object in space, the unit does not deform accordingly, and the quality of the unit is not maintained. change. The Lagrangian method is usually used to solve the problem of deformation of the Lagrange unit of a normal mass unit, which can accurately describe the displacement, deformation, stress, and so on.

Consider a motion system where a mass point is located at $B$ at the initial time $t=0$, and at a fixed Cartesian coordinate system, its coordinates are X. After time t, the particle moves to position b, and the coordinate in the same Cartesian coordinate system is. Lagrange describes incremental methods available.

The standard process of differential equations can be solved, but when the matrix order is high, the cost of using standard methods is very expensive. The nonlinear dynamic response of the finite element system is divided into two categories: direct integration method and modal superposition method. The direct integration method is solved in the time domain, the modal superposition method is solved in the frequency domain, and the collision problem is suitable for the former.

\section{Direct integration method}

The direct integration method is divided into explicit time integration method and implicit time integration method. If the discrete equation is first solved for the displacement vector $\mathrm{x}$, and then the speed vector and the acceleration vector are solved for the implicit algorithm, it can be seen that when solving the displacement vector The overall stiffness matrix must be decomposed and inverted. In the explicit method, due to the use of the lumped mass matrix $\mathrm{M}$, the solution of the equations of motion is uncoupled. There is no need to reorganize the overall stiffness matrix, nor does it need to perform matrix decomposition and seeking. Inverse, thus greatly saving storage space and computing time. The disadvantage is the strict limitation of the time step. Compared with the implicit time integration method, the integration time step required by the conditional stability of the explicit method must be less than the minimum natural period of the grid, and approximately 1 of the implicit integration time step, but the matrix decomposition is not required during each step of the calculation. The implicit method is unconditionally stable and has nothing to do with the time step of the integration. However, the stiffness matrix must be decomposed multiple times to affect the computational efficiency. In fact, in order to analyze the highly nonlinear dynamic response problems such as collisions, in order to ensure the accuracy of the calculation, no matter what method is used, the integration time step must be made very small. It can be seen that the explicit method is much superior to the implicit method to solve the problem of high transient non-linearity.

$$
x_{i}(i=1,2,3)
$$




$$
x_{i}=x_{i}\left(X_{a}, t\right)
$$

When a solid or structural surface is subjected to a dynamic load, the movements of particles in each part of the object cannot occur at the same time, and they need to go through a propagation process from the local disturbance zone to the undisturbed zone. The stress and deformation caused by the external load will be in the form of waves. Spread out. When the external load disturbance is weak, the elastic wave is generated. When the disturbance is strong, the plastic wave is generated. The stress will reach or exceed the initial yield stress of the material, and the crack may be caused along the propagation direction of the wave.

\section{Finite Element Applications}

Using finite element method or boundary element method to solve the contact problem has become the current mainstream, the basic point of these methods is the structure of the discretion, only the mathematical parameters on the node as the solution object, in the calculation model how to assume contact force on the contact surface Passing is the primary issue. One approach is to divide the contact surfaces of the two contact bodies into the same grid so that the node coordinates are in one-to-one correspondence to form a one-to-one corresponding node pair. Assuming that the contact force is transmitted through the pair of nodes, whether the local area on the contact surface has only normal contact and does not move tangentially, or whether there is tangential movement or no contact after deformation, the contact state is judged by the node pair. In the initial research of finite element solving contact problems, the node pair is the main or only mathematical model for a long time. It is intuitive, simple and easy to program, but when the contact surface is a complex surface, the grid nodes correspond one to one. The reason why it is not easy to do this is that when the friction slip is considered, the asymmetry of the final control equation brings great difficulties to the solution. The mathematical model of the node pair cannot asymmetry the equation. Therefore, the large-scale engineering is solved. Problems caused great trouble. Another mathematical model divides the two contact bodies into a master and a passive. The mathematical model is that a node in the active grid is in contact with any point on the surface of the passive contact (not necessarily a mesh node). This model is called a point surface model. The advantage of this model is that the contact body can be meshed according to its own conditions. After being processed, the final control equation can become symmetrical even if the friction slip condition is considered.
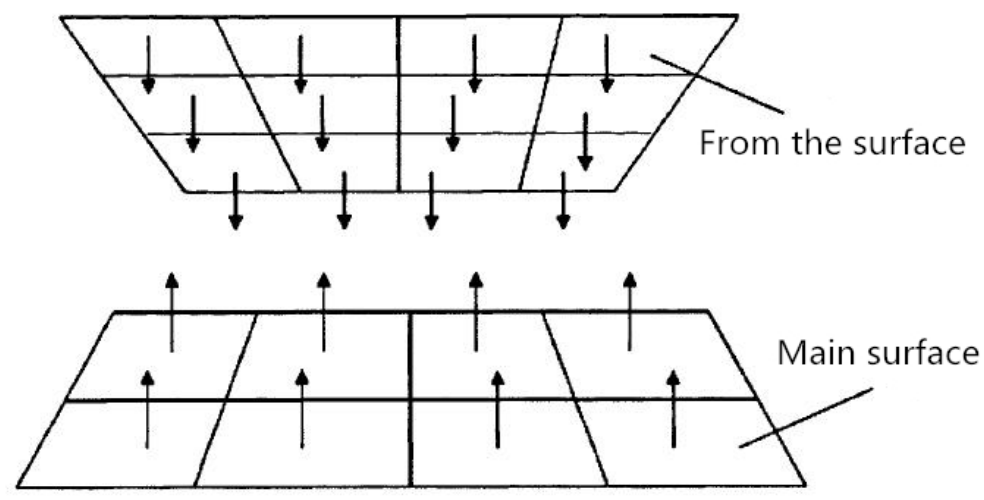

Figure 1 Master-slave surface contact

The latter (master-slave algorithm) is used for contact search when calculating the contact problem, and the contact force is calculated using the symmetric penalty function method. The principle is as follows: At each time step, it first checks whether each slave node penetrates the main plane. If there is no penetration, it does nothing to the slave node: if it penetrates, it is introduced between the slave node and the main plane that is penetrated. A large interface contact force, the size of which is proportional to the penetration depth and the stiffness of the main surface, is called the penalty 
function value. Its mathematical significance is equivalent to placing a normal spring between the slave node and the penetrated main face to limit the penetration of the slave node from the main face. Symmetric penalty function method is to process nodes on each main surface at the same time. The algorithm is the same as the slave node. The symmetry penalty function method is simple in programming and seldom arouses the hourglass effect of the grid, and there is no noise. This is due to the fact that the algorithm has symmetry and the momentum is conservative and does not require collision and release conditions. The size of the penalty function method is limited by stability. If significant penetration occurs in the calculation, the penalty function value can be enlarged or the time step can be reduced to adjust. The symmetric penalty function is used to cycle through the slave node and the main surface node at each time step, and the algorithm is the same.

Another problem with contact calculation is the law of friction. The reality is that the coefficient of friction depends not only on the material of the contact but also on the smoothness of the contact surface, the processing of the material, the lubrication conditions of the contact surface, and the surface pressure. Even if the same contact has a sliding relationship with the load, the friction coefficient will change. The relationship is nonlinear. At present, most of the literature still use the Coulomb friction law, that is, the friction coefficient is constant during the solution process, and generally separates the static and dynamic friction coefficients. Ls. Coulomb's law is still used when Dvina solves the contact friction force. Consider the static friction coefficient and obtain the contact friction force through the normal contact force.

$$
\left\{\begin{array}{l}
F^{n+1}=F^{n},\left|F^{n}\right| \leq F_{y} \\
F^{n+1}=F_{y} F^{n} /\left|F^{n}\right|,\left|F^{n}\right| \geq F_{y}
\end{array}\right.
$$

In our simulation of neoplastic flow problems, there is no standard cell shape to refer to. The boundary of the current tetrahedral mesh is just an expression of the object boundary. In order to avoid excessive smooth optimization work, additional methods must be used to handle the boundary. No matter what simulation method is used, the boundary problem is one of the most important and difficult problems in graphics. In the Euler method for fluid simulation based on finite element meshes, many researchers have proposed many methods to represent the surface of the fluid. For example, Euler method and particle spring model particle method are combined to track the fluid surface. High-density surface particles are used in the finite element method. According to the original low-quality grid, a new high-quality grid is generated according to the method of re-grid described above.

The solution process of the finite element discrete equations is a Lagrangian direct implicit integration method with variable time steps. In order to determine when to reduce the time step, you must check whether there are sharp changes in the edges of each tetrahedron. When it is detected at a time step that an edge change exceeds a certain threshold, the state before this time step must be returned and the time step is halved. The extra damping caused by volume bundling and large plastic strain makes it difficult to detect instability only by the length of the edge, and the time step must be reduced whenever sudden acceleration occurs. If the problem of instability does not occur after several successful simultaneous calculations, the time step can be doubled to reduce the solution time. The Lagrangian implicit algorithm is unconditionally stable, although the time step size does not affect the solution stability but affects the solution accuracy.

\section{Conclusion}

The finite element method has become the main numerical method for solving engineering problems in computational mechanics. However, with the extension of its application scope, some of its inherent defects also stand out. In the field simulations involving large deformations such as press forming and high-speed collisions, the finite element mesh based on the Lagrangian method may cause severe distortions, and even make the Jacobean of the element negative, not only in calculations. The need for grid reconstruction, and seriously affect the accuracy of the solution; for high-speed impact and other dynamic problems, the step size of the explicit time integration depends on the 
minimum size of the finite element mesh, so the distortion of the grid will make the time integration step When the length is too small, the computational workload is greatly increased. As for the dynamic expansion of cracks, the direction of crack propagation cannot be determined in advance. Therefore, it is necessary to continuously re-divide the grid to simulate the dynamic expansion process of cracks in the calculation process. Since the finite element approximation is based on a mesh, it is difficult to handle discontinuities and large deformations that are inconsistent with the original mesh lines. Grid reconstruction is not only computationally expensive, but also impairs the accuracy of the calculation. In view of this kind of defect, in recent years, the finite element method adopts a point-based approximation, which can eliminate the mesh completely or partially, does not require the initial division and reconstruction of the mesh, not only can guarantee the accuracy of calculation, but also can greatly reduce The difficulty of the calculation. Therefore, there has been a small amount of research in computer graphics. However, since the current finite element approximation does not generally have an analytical expression, the computational load is large and exceeds the traditional finite element method. In addition, finite element approximations are mostly fitting, and therefore it is difficult to handle displacement boundaries. Lagrange multiplier method is used for processing.

The finite element method does not need to generate a grid in the numerical calculation, but constructs discrete control equations of the interpolation function according to some arbitrary distributed coordinate points, and it is convenient to simulate various complex shapes of the flow field. The finite element method has the convenience that the limited element method does not have in the computer graphics simulation of the point model. Because it reads, changes, calculates, and displays point data, it is point data. It does not have to be converted to generate grids and data extraction can save a lot of time. It can be predicted that as grid less methods mature, computing time With respect to the continuous reduction of the finite element method, the application in computer simulation will be more and more bright prospects.

\section{References}

[1]. Wu Yongli, et al. Comparison of some solutions of elastic-plastic finite element. Chinese Journal of Solid Mechanics,Vol. 13 (1982) No. 6, p. 565-570.

[2]. Qiao Duan et al, et al. Full elastic-plastic finite element method and its application. Journal of Beijing Institute of Iron and Steel,Vol. 23 (1985) No. 9, p. 104-113

[3]. Wei Zujian et al, et al. Uniform tangent modulus in nonlinear elasticity and elastic-plastic finite element analysis. Journal of Applied Mechanics,Vol. 19 (1992) No.2, p. 70-76

[4]. Aravind Kalaiah and Amitabh Varshney, et al. Modeling and rendering points with local geometry. IEEE Transactions on Visualization and Computer Graphics,Vol. 34 (2003) No.11, p. 30-42.

[5]. Renato Pajarola, et al. Efficient level-of-details for point based rendering. In Proceedings IASTED Invernational Conference on Computer Graphics and Imaging,Vol. 39 (2003) No.2, p.

[6]. Renato Pajarola, Miguel Sainz, and Patrick Guidotti, et al. Object-space point blending and splatting. In ACM SIGGRAPH Sketches\&Applications Catalo-gue,Vol. 16 (2003) No.6, p.

[7]. Renato Pajarola, Miguel Sainz, and Patrick Guidotti. Confetti, et al. Object-space point blending and splatting. IEEE Transactions on Visualization and Computer Graphics,Vol. 16 (2004) No. 5, p. 598-60.

[8]. Wenland H. Piecewise polynomial, et al. positive definite and compactly supported radial basis functions of minimal degree. Advances in Computational Mathematics,Vol. 23 (1995) No. 3, p.389-396. 